\title{
Cerebral motor control in patients with gliomas around the central sulcus studied with spatially filtered magnetoencephalography
}

\author{
M Taniguchi, A Kato, H Ninomiya, M Hirata, D Cheyne, S E Robinson, M Maruno, Y Saitoh, \\ H Kishima, T Yoshimine
}

J Neurol Neurosurg Psychiatry 2004;75:466-471. doi: 10.1136/jnnp.2002.01834

See end of article for authors' affiliations

.....................

Correspondence to:

Dr Masaaki Taniguchi,

Department of

Neurosurgery, E6, Osaka

University Medical School,

2-2 Yamadaoka, Suita,

Osaka 565-0871, Japan; mtani@

nsurg.med.osaka-u.ac.jp

Received 16 August 2002 In final revised form 23 June 2003

Accepted 24 June 2003
Objective: Application of spatially filtered magnetoencephalography (MEG) to investigate changes in the mechanism of cerebral motor control in patients with tumours around the central sulcus.

Methods: MEG records were made during a repetitive hand grasping task in six patients with gliomas around the central sulcus and in four control subjects. Power decreases in the $\alpha(8-13 \mathrm{~Hz}), \beta(13-30 \mathrm{~Hz})$, and low $\gamma$ bands $(30-50 \mathrm{~Hz}$ ) during the motor tasks (event related desynchronisation, ERD) were analysed statistically with synthetic aperture magnetometry. The tomography of ERD was superimposed on the individual's magnetic resonance image.

Results: $\beta$ ERD was consistently localised to the contralateral primary sensorimotor cortex $(\mathrm{MI} / \mathrm{SI})$ in control subjects, whereas the $\alpha$ and low $\gamma$ ERD showed considerable intersubject variability. $\beta$ ERD in patients during non-affected side hand movement was also localised to the contralateral $\mathrm{MI} / \mathrm{SI}$, but exclusively to the ipsilateral hemisphere during affected side hand movement.

Conclusions: The altered pattern of ERD in the patient group during affected side hand movement suggests recruitment of diverse motor areas, especially the ipsilateral $\mathrm{MI} / \mathrm{SI}$, which may be required for the effective movement of the affected hand.
1 nitial impairment of motor function after ischaemic or traumatic/surgical insults often recovers to a certain degree with intensive physiotherapy. In slowly progressive tumours, impairment of motor function does not become apparent until a certain area of the motor cortex is involved. ${ }^{1}$ Clinical observations of patients suggest that functional remodelling in the brain is taking place during recovery from the acute insult or during the gradual growth of the tumour. For surgical treatment of tumours involving the motor areas, elucidation of this remodelling is of great importance for presurgical planning and for programming effective rehabilitation after initial treatment.

Various different studies with positron emission tomography $(\mathrm{PET})^{23}$ and functional magnetic resonance imaging $(\mathrm{fMRI})^{4-6}$ in patients with diverse lesions affecting cerebral motor control have shown altered activation patterns in the motor areas. Those studies mainly depict haemodynamic changes generated during the motor task. High resolution electroencephalography ${ }^{7}$ was capable of capturing neural activation directly, but the results were only demonstrable on gross topography.

Magnetoencephalography (MEG) has been employed o demonstrate neural activation directly with enhanced spatial resolution. Using a currently available signal processing technique-synthetic aperture magnetometry (SAM) - multiple simultaneously active sources became subjects for the analysis. ${ }^{8}$ Combined with statistical comparison of the power of each source, ${ }^{9}$ a differential study between two arbitrarily chosen states became possible, which is much easier to do than the time locked motor task. ${ }^{10}{ }^{11}$

A power decrease in background brain activity during a motor task was first demonstrated in EEG studies and was termed "event related desynchronisation" (ERD). ${ }^{12}$ This was evident in the primary sensorimotor area and was considered to be a representation of the active cortex during the motor task. ${ }^{13}{ }^{14}$ In the present study, ERD on $\alpha, \beta$, and low $\gamma$ bands during a grasping task was studied in patients with intrinsic brain tumours around the central sulcus.

\section{METHODS}

Subjects

Six patients (ranging in age from 36 to 60 years) with gliomas around the central sulcus and six control subjects (age range 33 to 47 years) gave their informed consent for the experimental procedures and participated in the MEG study. Five patients and all control subjects were right handed and one was left handed according to the modified Oldfield's inventory. ${ }^{15}$ Four patients had a tumour in their dominant hemisphere. Clinical data on the patients are given in table 1 . Subject 1 was studied at one month after tumour removal while he was gradually recovering from his right sided weakness (from 3/5 immediately after surgery to $4 / 5$ at the time of MEG recording, assessed by manual muscle testing). Subject 5 had a five months history of fine movement disorder before the study. Others had experienced no motor complications, and all were capable of carrying out the motor task.

\section{Motor task and data acquisition}

A helmet shaped 64 channel SQUID array (NeuroSQUID Model 100, CTF Systems Inc, Port Coquitlam, Canada) was used for MEG data acquisition. Details of the motor task have been described previously. ${ }^{10}$ In brief, subjects were instructed to undertake a trial consisting of six sessions of repetitive hand grasping (not a tonic sustained grasp) of either hand

Abbreviations: ERD, event related desynchronisation; fMRI, functional magnetic resonance imaging; MEG, magnetoencephalography; $\mathrm{MI}$, primary motor cortex; $\mathrm{Ml} / \mathrm{Sl}$, sensorimotor cortex; $\mathrm{PET}$, positron emission tomography; SAM, synthetic aperture magnetometry; SI, primary sensory cortex 


\begin{tabular}{|c|c|c|c|c|}
\hline Patient No & Sex/age (years) & Handedness & Diagnosis/tumour location & Symptom \\
\hline 1 & Male/57 & Right & $\begin{array}{l}\text { Glioblastoma/L superior frontal- } \\
\text { cingulate gyrus }\end{array}$ & $\begin{array}{l}R \text { weakness ( } 4 / 5 \text { according } \\
\text { to } M M T \text {, one month after } \\
\text { tumour removal), seizure }\end{array}$ \\
\hline 2 & Male/36 & Right & $\begin{array}{l}\text { Astrocytoma/R superior frontal } \\
\text { gyrus }\end{array}$ & $\begin{array}{l}\text { No neurological deficit } \\
\text { (diagnosed incidentally) }\end{array}$ \\
\hline 3 & Male/49 & Right & $\begin{array}{l}\text { Oligoastrocytoma/R pre- and } \\
\text { post-central gyrus }\end{array}$ & Psychomotor seizure \\
\hline 4 & Male/38 & Right & Astrocytoma/L precentral gyrus & Seizure \\
\hline 5 & Female/51 & Right & Glioblastoma/L postcentral gyrus & $\begin{array}{l}\text { R weakness (five months' } \\
\text { history of fine movement } \\
\text { disorder), seizure }\end{array}$ \\
\hline 6 & Male/60 & Left & Glioblastoma/R postcentral gyrus & Dysaesthesia in left thumb \\
\hline
\end{tabular}

for 10 seconds after 10 seconds of rest, keeping their eyes closed. The beginning and end of the movement was signalled to the subject by the investigator. Movements were monitored on video throughout the recording. All subjects practised the task before data acquisition to learn to grasp the hand appropriately without excessive movement of the forearm. The grasping rate was about $2 \mathrm{~Hz}$, and absence of apparent mirror movement was confirmed on EMG or on inspection. The MEG data were acquired on trigger at the very end of the 10 seconds of grasping with a $625 \mathrm{~Hz}$ sampling rate and on-line low pass filtering of $200 \mathrm{~Hz}$. Individual anatomical MRI datasets were acquired using $1.5 \mathrm{~T}$ imaging systems (Magnetom Impact, Siemens, Erlangen, Germany). For all measurements, fiducial skin markers were placed on the subject's nasion and at bilateral preauricular points to establish common coordinate systems for MRI and MEG for subsequent superimposition of the MEG results on the individual magnetic resonance images.

\section{MEG analysis}

Detail of the SAM algorithm have been described previously. ${ }^{8} 1617$ SAM is a spatial filtering technique based on the adaptive beamformer theory. It estimates source activity at each selected voxel within the region of interest. ${ }^{18} \mathrm{~A}$ volumetric image of root mean squared source activity in $\alpha, \beta$, and low $\gamma$ bands with $2 \mathrm{~mm}$ voxel resolution is generated for time intervals of -20 to -15 and -5 to 0 seconds relative to trigger onset as control and active states, respectively. The statistical imaging is computed subsequently by comparing the power of both states on a single voxel basis using the Student $t$ test. Only voxels displaying peak signal changes within each trial are displayed on the individual magnetic resonance images. Images with a peak $t$ value less than 2.5 or ERD distributed evenly over the hemisphere were excluded. To investigate the left-right difference in the basic rhythm, the raw MEG data in the control state were fast Fourier transformed. The $\beta$ band power values of channels covering the sensorimotor cortex in both hemispheres were superimposed and compared.

\section{Direct cortical stimulation}

In two subjects, cerebral cortex adjacent to the lesion was stimulated at the time of surgery. The stimulation was conducted with the patient awake. A bipolar probe with a current of $10 \mathrm{mV}$ at $50 \mathrm{~Hz}$ was used. The motor responses were confirmed either on inspection by the trained investigator or by patient's self report.

\section{RESULTS}

Monitoring of the movement during the recording revealed an almost congruent grasping rate of about $2 \mathrm{~Hz}$ in both the control group and the patient group, except for subject 5 who

Table 2 Areas displaying most significant power changes in the $\alpha, \beta$, and low $\gamma$ bands during each motor task in the normal subjects $(n=4)$

\begin{tabular}{|c|c|c|c|c|}
\hline \multirow[b]{2}{*}{ Side of movement } & & \multicolumn{3}{|c|}{ Number of trials / $t$ maximum of each trial } \\
\hline & & $\alpha(8-13 \mathrm{~Hz})$ & $\beta(13-30 \mathrm{~Hz})$ & Low $\gamma(30-50 \mathrm{~Hz})$ \\
\hline \multirow[t]{3}{*}{$\begin{array}{l}\text { Dominant hand } \\
\text { movement }\end{array}$} & $\begin{array}{l}\text { Contralateral } \mathrm{MI} / \mathrm{SI} \\
\text { Contralateral superior parietal } \\
\text { lobule }\end{array}$ & $1 / 5.65$ & $3 / 2.89$ to 4.45 & $\begin{array}{l}3 / 2.56 \text { to } 3.50 \\
1 / 2.56\end{array}$ \\
\hline & $\begin{array}{l}\text { Ipsilateral } \mathrm{MI} / \mathrm{SI} \\
\text { Ipsilateral superior parietal } \\
\text { lobule }\end{array}$ & $\begin{array}{l}1 / 2.9 \\
2 / 2.92 \text { to } 3.6\end{array}$ & $\begin{array}{l}1 / 3.0 \\
1 / 2.89\end{array}$ & \\
\hline & $\begin{array}{l}\text { Ipsilateral inferior parietal } \\
\text { lobule }\end{array}$ & & & $1 / 2.73$ \\
\hline \multirow{5}{*}{$\begin{array}{l}\text { Non-dominant hand } \\
\text { movement }\end{array}$} & Contralateral $\mathrm{MI} / \mathrm{SI}$ & $1 / 2.93$ & $4 / 2.85$ to 4.99 & $3 / 2.7$ to 3.60 \\
\hline & $\begin{array}{l}\text { Contralateral superior parietal } \\
\text { lobule }\end{array}$ & $1 / 2.93$ & $1 / 2.85$ & \\
\hline & $\begin{array}{l}\text { Contralateral inferior parietal } \\
\text { lobule }\end{array}$ & $1 / 4.0$ & $1 / 4.05$ & \\
\hline & $\begin{array}{l}\text { Contralateral frontal operculum } \\
\text { Ipsilateral } \mathrm{MI} / \mathrm{SI} \\
\text { Ipsilateral superior parietal } \\
\text { lobule }\end{array}$ & $\begin{array}{l}1 / 5.01 \\
1 / 5.01\end{array}$ & $\begin{array}{l}1 / 2.85 \\
2 / 2.85 \text { to } 4.99\end{array}$ & $1 / 3.56$ \\
\hline & $\begin{array}{l}\text { Ipsilateral occipital lobe } \\
\text { Ipsilateral superior temporal } \\
\text { gyrus }\end{array}$ & $\begin{array}{l}1 / 4.22 \\
1 / 2.93\end{array}$ & & \\
\hline
\end{tabular}


Normal subject

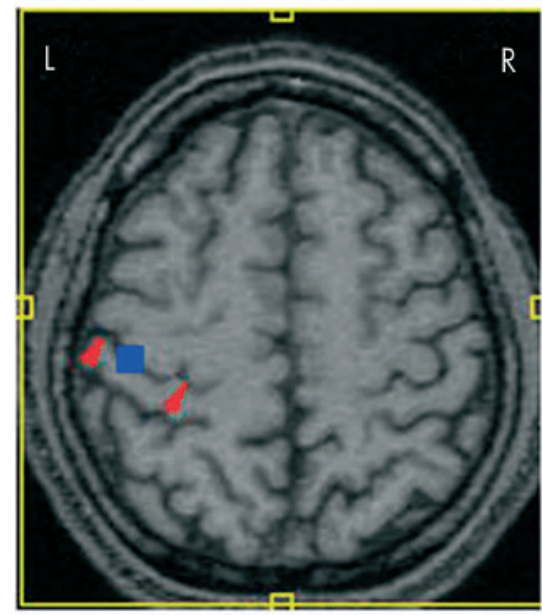

Right hand movement

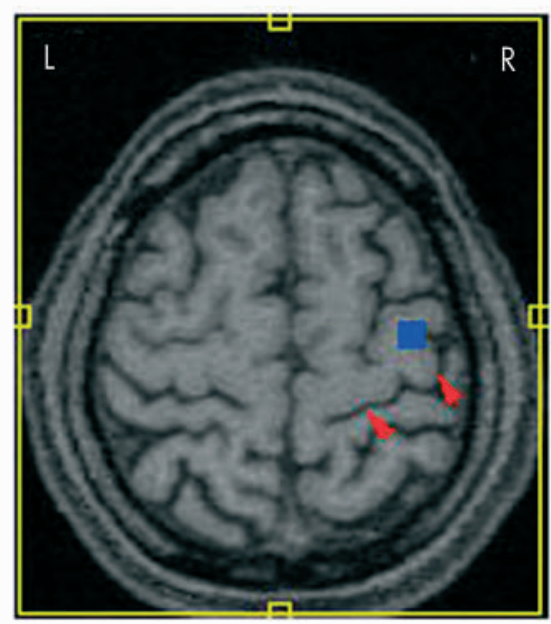

Left hand movement

Figure 1 Voxels displaying most significant event related desynchronisation (ERD) in the $\beta$ band $(13-30 \mathrm{~Hz})$ during right and left hand grasping in a control subject are displayed in blue superimposed on the subjects' anatomical magnetic resonance image. Arrowheads indicate the central sulcus. Note the ERD on the contralateral sensorimotor cortex in the control subject.

had weak motor palsy resulting in a decreased grasping rate of about $1 \mathrm{~Hz}$.

\section{Control subjects}

Two subjects' results were excluded from the analysis as the voxels with the highest $t$ value were distributed evenly over the entire hemisphere. In the other four subjects, the $t$ value ranged from 2.56 to 5.65 (table 2). A consistent ERD in the sensorimortor cortex (MI/SI) contralateral to the hand movement was observed in the $\beta$ band (fig l) except in one subject, who showed ipsilateral SI activity during dominant hand grasping. Those ERD were almost congruent with the "knob" ${ }^{\prime 19}$ or "omega shaped structure" ${ }^{\prime 20}$ which is considered to be the hand representative area. Additional $\beta$ ERD was observed on the ipsilateral superior parietal lobule during dominant hand movement (one subject) and on the ipsilateral MI/SI (two subjects), the contralateral inferior parietal lobule (one subject), and the superior parietal lobule and frontal operculum (one subject) during non-dominant hand movement (fig 2).

Low gamma ERD was observed in the contralateral MI/SI during dominant and non-dominant hand movement (three subjects). In contrast, $\alpha$ ERD was observed over diverse regions of both cerebral hemispheres.

\section{Patients}

Four patients had $\gamma$ ERDs with $t$ values less than 2.5 and were excluded from subsequent analysis. In other frequency bands, the $t$ value ranged from 2.54 to 6.78 (table 3 ). For hand movement on the non-affected side, the $\beta$ ERD was observed on contralateral MI/SI and in the inferior parietal lobule (three and four subjects, respectively). For hand movement on the affected side the $\beta$ ERD was observed on ipsilateral MI/SI lateral to the assumed hand representative area, in the lateral premotor area, and in the inferior parietal lobule (four, two, and one subjects, respectively) (figs 3 and 4).

The low $\gamma$ ERD was observed in the contralateral MI/SI and the premotor area during non-affected hand movement (two subjects each) and in the ipsilateral MI/SI during affected hand movement (three subjects). The $\alpha$ ERD was observed in diverse regions without any strong consistency.

\section{$\beta$ Band power}

Left-right difference in $\beta$ band power in the resting state was negligible in the control subjects (figs 5 and 6). In the patient group, attenuation of the $\beta$ band power was evident, especially in the sensors covering the central region in the affected hemisphere (statistically significant for sensors 23, 24,33 , and 34 by two tailed Student $t$ test (fig 6)).

\section{Cortical stimulation}

In both subjects studied, weak muscular contractions in the contralateral upper extremity were elicited with the stimulation of the cerebral cortex adjacent to the lesion around the central sulcus.
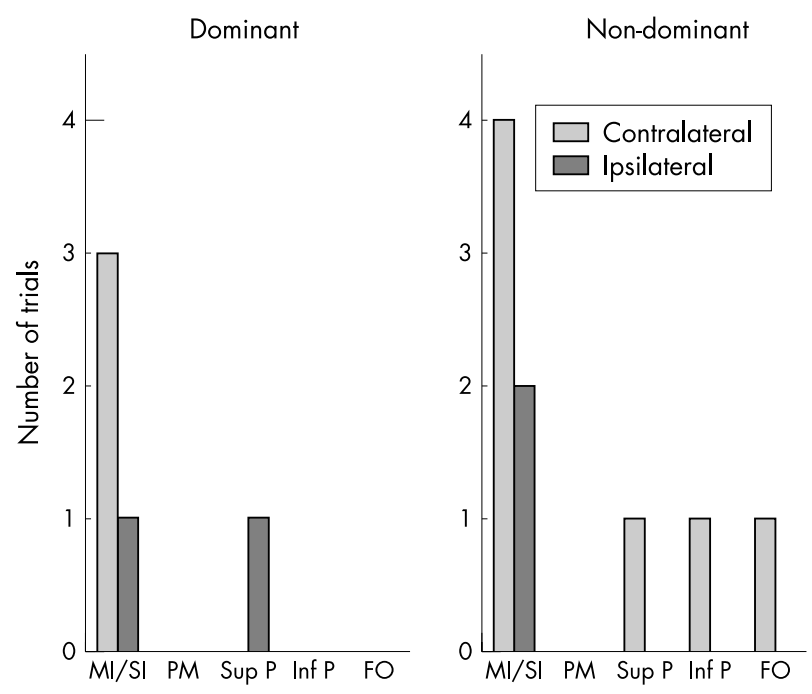

Figure 2 Number of trials showing $\beta$ event related desynchronisation (ERD) on motor areas for control subjects during dominant hand movement (left) and non-dominant hand movement (right). FO, frontal operculum; Inf $\mathrm{P}$, inferior parietal lobule; $\mathrm{MI} / \mathrm{SI}$, sensorimotor area; $\mathrm{PM}$, premotor area; Sup P, superior parietal lobule. 


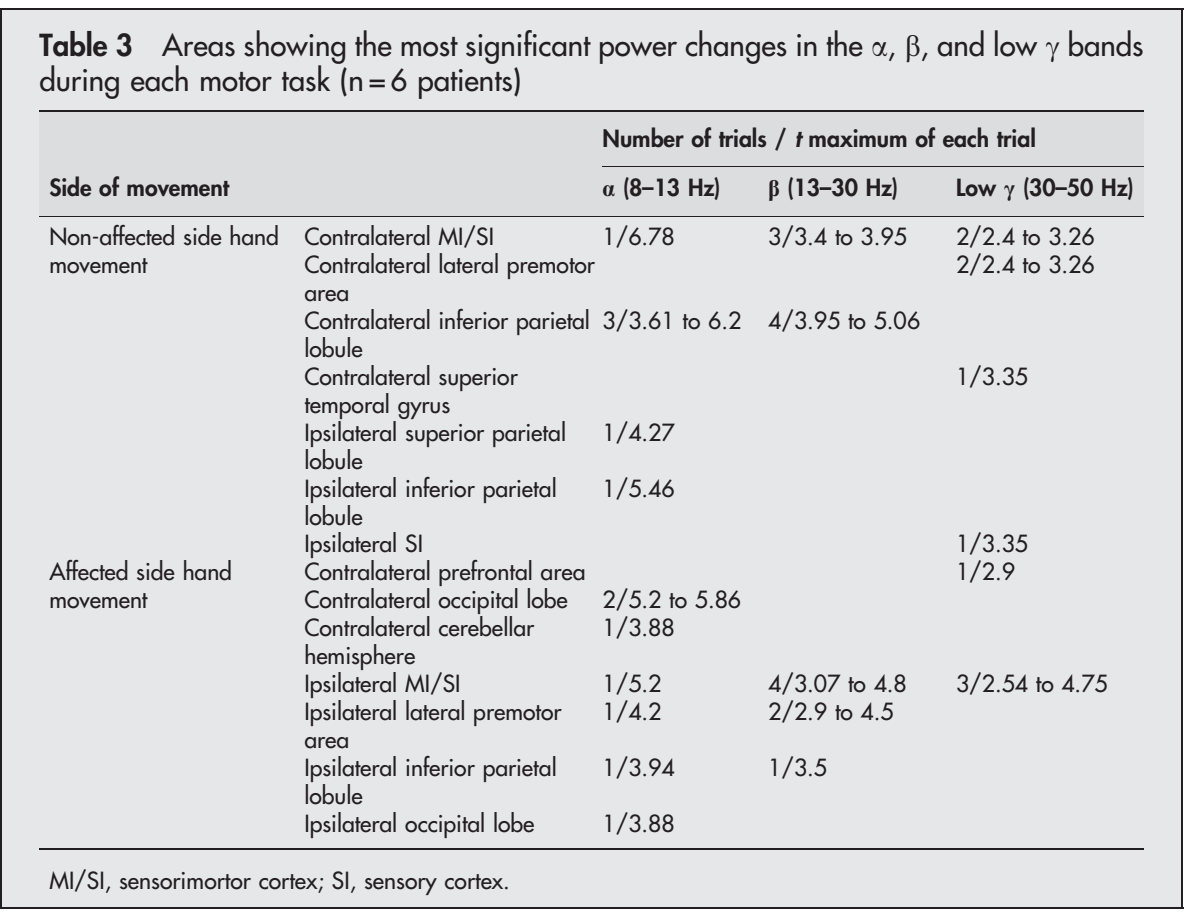

\section{DISCUSSION}

In control subjects, the $\beta$ ERD was consistently observed in the contralateral MI/SI adjacent to the so called "knob" or "omega shaped structure", ${ }^{19} 20$ as in previous studies. ${ }^{10}{ }^{14} \mathrm{In}$ contrast, the $\alpha$ ERD showed considerable intersubject variability. Compared with the previous studies, ${ }^{21}$ the task employed in the present one was repetitive grasping over a fixed time interval; this was not time locked, so both the planning and the execution and proprioceptic feedback phases of each movement were included in the analysis. This, and the broad classical frequency width applied for each band being analysed, may thus have blurred the phase and frequency specific ERD and resulted in the lack of consistent $\alpha$ ERD. This may also explain why movement related synchronisation in the low $\gamma$ band $^{22}$ was only observed in five of 12 trials. The low $\gamma$ ERD was also less concentrated in primary motor areas, appeared to be less constrained to the primary hand representation area, and showed less contralateral dominance. Based on a clearer localisation in the control group, our subsequent analysis therefore concentrated on the $\beta$ band ERD.

The most prominent finding in our study was the lack of $\beta$ ERD in the MI/SI contralateral to the hand movement on the affected side in the patients (figs 2 and 4). This does not necessarily mean a lack of activity in the MI/SI, as ERD is a reflection of the modulation on basic rhythm by a motor event. Thus if the basic rhythm were suppressed in the resting state owing to the presence of the tumour and the surrounding oedema, the ERD would not become apparent. The $\beta$ band power attenuation in sensors on the affected side in the resting state, in comparison with the non-affected side (as shown in figs 5 and 6), would provide evidence for the

Patient 4: right hand movement
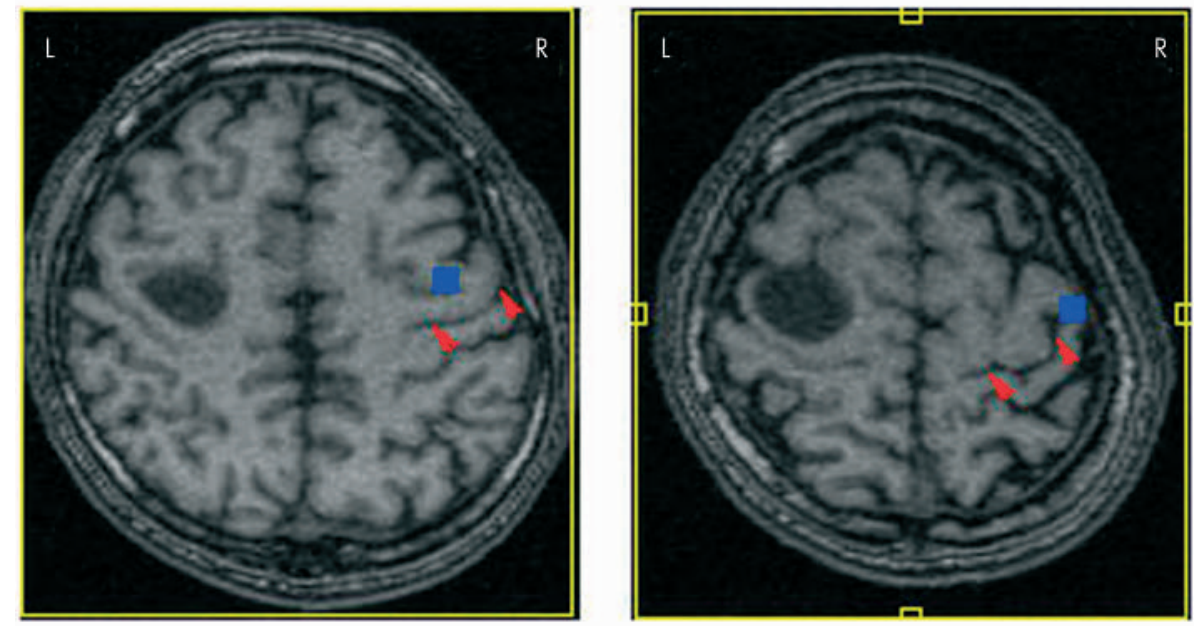

Figure $3 \quad \beta$ Band event related desynchronisation (ERD) in patient 4 during right hand grasping. Arrowheads indicate the central sulcus. Note the exclusive ipsilateral multiple ERDs in the patients during the affected side hand movement. 

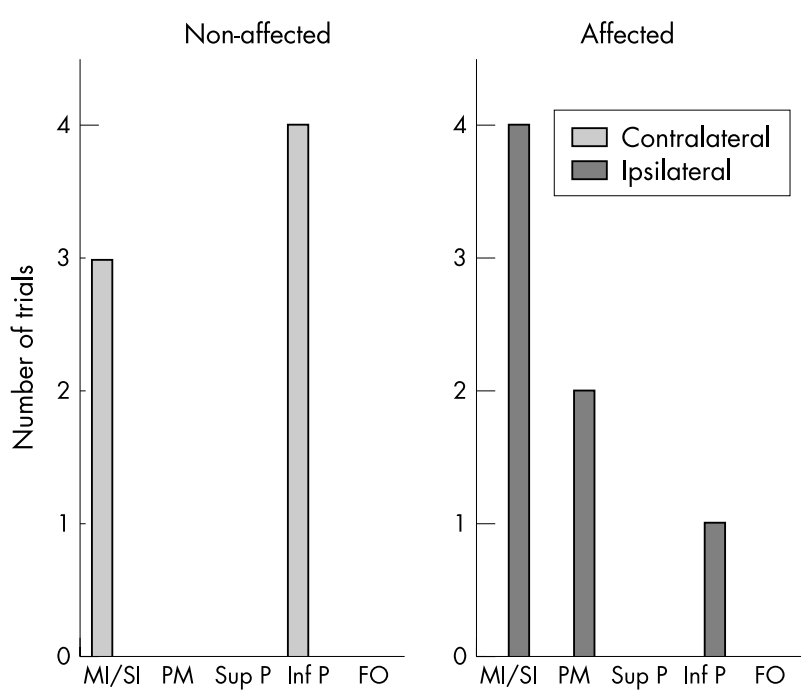

Figure 4 Number of trials showing $\beta$ band event related desynchronisation (ERD) in motor areas for patients during non-affected hand movement (left) and affected hand movement (right). Note the exclusive ipsilateral sensorimotor area ERD for affected side movement. $\mathrm{FO}$, frontal operculum; Inf $\mathrm{P}$, inferior parietal lobule; $\mathrm{MI} / \mathrm{SI}$, sensorimotor area; PM, premotor area; Sup P, superior parietal lobule.

basic rhythm alteration in the tumour bearing hemisphere, making the above mentioned hypothesis plausible. Nevertheless, the ipsilateral ERD during affected hand movement would suggest that considerable mobilisation of the ipsilateral motor areas is mandatory to maintain appropriate motor function. This suggests that the affected motor cortex does not function fully in the normal range even at the stage when the motor impairment is not apparent at all, as was the case in most of the patients studied. The fact that ipsilateral ERD was also observed in subjects in whom intraoperative cortical stimulation showed motor responses around the contralateral motor area suggests that one strategy preferentially employed by the brain in the early stage of motor impairment is recruitment of diverse motor areas, especially the ipsilateral MI/SI.

Another striking feature was the inferior parietal cortex ERD in four of six patients during non-affected hand movement. As this region is considered to become active during motor attention, ${ }^{23}$ the results suggest that more attention was needed in those patients. That is likely to have been the case as the task employed was repetitive grasping, which probably requires repeated disengagement and switching of the focus of motor attention from one movement to another. Inferior parietal cortex ERD might also have been present in the contralateral hemisphere during affected side movement, but for the same reason that the ERD of MI/SI does not become apparent owing to the reduced basic $\beta$ band power, this ERD might also have been masked. Whether this is the result of any form of motor plasticity is not clear and would need further exploration.

MEG depicts neuronal activity directly, with superior temporal and spatial resolution. ${ }^{24}$ Recording of the motor related field, however, is based on time locked motor tasks, ${ }^{25}{ }^{26}$ which require practice to obtain a consistent result, even in control subjects. Such complex tasks for the patient would result in high intertrial variability, as various aspects of a motor movement-for example, rate and strength of a movement-would affect the result considerably. ${ }^{27}{ }^{28}$ As the application of SAM source analysis does not require identification of peak amplitudes in the averaged response for discrete movements and dipole modelling, arbitrarily

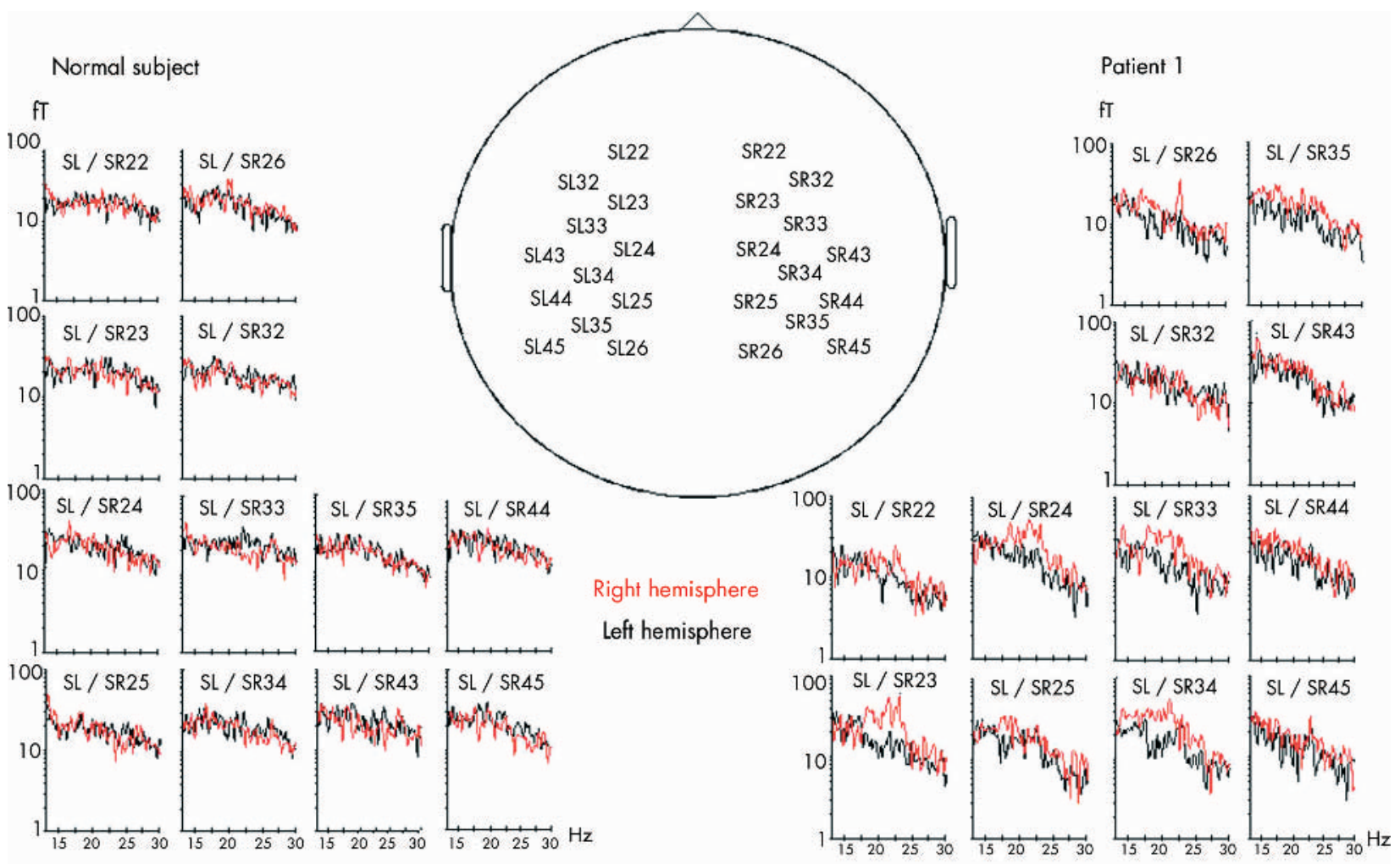

Figure 5 Superimposition of the $\beta$ band power during resting stage in the sensors covering the central region in a control subject (left) and patient 1 (right). Signal power changes in sensors over the right and left hemispheres are indicated in red and black, respectively. Note the basic power attenuation in sensors $23,24,25,33,34$, and 35 covering left (affected side) central region in patient 1 . 


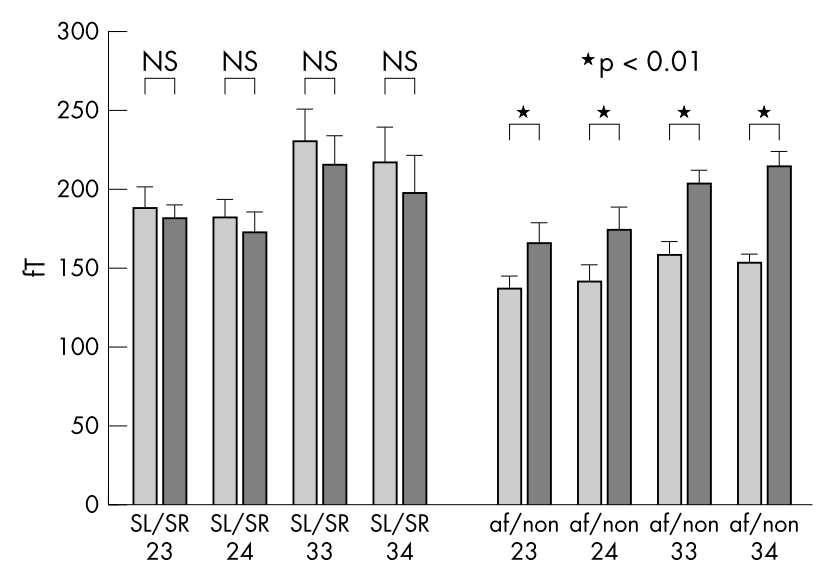

Figure 6 Bar graph indicating $\beta$ band power (fT) of representative channels covering the sensorimotor cortex for normal subjects and for the patient group. Values are means, error bars $=S D$. Note the significant difference between channels over the affected and nonaffected hemispheres in the patient group. Though some differences between left and right channels in the normal subjects were demonstrated, these were not statistically significant. af, channels over the affected hemisphere; non, channels over the non-affected hemisphere; SL, MEG sensors on left hemisphere; SR, MEG sensors on right hemisphere.

paced tasks such as repetitive grasping are applicable. The grasping task employed in this study was simple, so little effect of differences in the rate and strength of movement could be expected on the activation pattern. All subjects were instructed to grasp the hand weakly at a constant rate, which was confirmed on the monitor during the recording. Thus differences in the appearance of each motor movement could not be accounted for the differences between the control and patient groups. Finally, based on our previous studies of movement related ERD, ${ }^{10}$ in the current study, we included voxels displaying an ERD with a pseudo $t$ value of 2.5 or greater. However, the use of the SAM pseudo $t \operatorname{score}^{29}$ makes direct statistical comparisons between experimental conditions and subject groups difficult and remains an area for further development.

\section{ACKNOWLEDGEMENTS}

This work was supported in part by grants-in-aid for scientific research from the Japanese Ministry of Health and Welfare, Nos $11470290,13770764,13470293$, and 14657342, and by the Osaka Medical Research Foundation for Incurable Diseases.

\section{Authors' affiliations}

M Taniguchi, A Kato, H Ninomiya, M Hirata, M Maruno, Y Saitoh, H Kishima, T Yoshimine, Departments of Neurosurgery, Osaka University Graduate School of Medicine, Osaka, Japan

D Cheyne, Hospital for Sick Children Research Institute, Toronto, Ontario, Canada

S E Robinson, CTF systems Inc, Port Coquitlam, British Columbia, Canada

Competing interests: none declared

\section{REFERENCES}

1 Frankel SA, German WJ. Glioblastoma multiforme: review of 219 cases with regard to natural history, pathology, diagnostic methods, and treatment. J Neurosurg 1956;13:489-503.
2 Chollet F, DiPiero V, Wise RJS, et al. The functional anatomy of motor recovery after stroke in humans: a study with positron emission tomography. Ann Neurol 1991;29:63-71.

3 Nelles G, Spiekermann G, Jueptner M, et al. Evolution of functional reorganization in hemiplegic stroke: a serial positron emission tomographic activation study. Ann Neurol 1999;46:901-9.

4 Cao Y, D'Olhaberriague L, Vikingstad EM, et al. Pilot study of functional MRI to assess cerebral activation of motor function after contralateral hemiparesis. Stroke 1998;29:112-22.

5 Cramer SC, Finklestein SP, Schaechter JD, et al. Activation of distinct motor cortex regions during ipsilateral and contralateral finger movements. J Neurophysiol 1999;81:383-7.

6 Fandino J, Kollias SS, Wieser HG, et al. Intraoperative validation of functional magnetic resonance imaging and cortical reorganization patterns in patients with brain tumors involving the primary motor cortex. J Neurosurg 1999;91:238-50.

7 Green JB, Bialy Y, Sora E, et al. High-resolution EEG in poststroke hemiparesis can identify ipsilateral generators during motor tasks. Stroke 1999;30:2659-65

8 Robinson SE. Method for functional brain imaging from magnetoencephalographic data by estimation of source signal-to-noise ratio. United States Patent Application 1998;09/138, 826 filed 24 August 1998.

9 Ishii R, Shinozaki K, Ukai S, et al. Medial prefrontal cortex generates frontal midline theta rhythm. Neuroreport 1999;10:675-9.

10 Taniguchi M, Kato A, Fujita N, et al. Movement-related desynchronization of the cerebral cortex studied with spatially filtered magnetoencephalography. Neuroimage 2000;12:298-306

11 Hirata M, Kato A, Taniguchi M, et al. Frequency-dependent spatial distribution of human somatosensory evoked neuromagnetic fields. Neurosci Lett 2002;318:73-6.

12 Pfurtscheller G. Central beta rhythm during sensorimotor activities in man. Electroencephalogr Clin Neurophysiol 1981;51:253-64.

13 Pfurtscheller G, Lopes da Silva FH. Event-related EEG/MEG synchronization and desynchronization: basic principles. Clin Neurophysiol 1999; 110:1842-57.

14 Salmelin R, Hämäläinen $M$, Kajola $M$, et al. Functional segregation of movement-related rhythmic activity in the human brain. Neuroimage 1995;2:237-43.

15 Oldfield $\mathbf{R}$. The assessment and analysis of handedness: the Edinburgh inventory. Neuropsychologia 1971;9:97-113.

16 Robinson SE, Rose DF. Current source image estimation by spatially filtered MEG. In: Hoke M, et al. eds. Biomagnetism: clinical aspects. Munster: Elsevier Science, 1992:761-5.

17 Robinson SE, Vrba J. Functional neuroimaging by synthetic aperture magnetometry (SAM). In: Yoshimoto T, Kotani M, Kuriki S, et al. eds. Recent advances in biomagnetism. Sendai: Tohoku University press, 1999:302-5

18 Widrow B, Stearns SD. Beamformers with superresolution: adaptive signal processing. Upper Saddle River: Prentice-Hall, 1985.

19 Yousry TA, Schmid UD, Alkhadhi H, et al. Localization of the motor hand area to a knob on the precentral gyrus. A new landmark. Brain 1997; 120:141-57.

20 Puce A, Constable RT, Luby ML, et al. Functional magnetic resonance imaging of sensory and motor cortex: comparison with electrophysiological localization. J Neurosurg 1995;83:262-70.

21 Pfurtscheller G, Neuper C, Berger J. Source localization using event-related desynchronization (ERD) within the alpha band. Brain Topogr 1994;6:269-75.

22 Pfurtscheller G, Neuper C, Kalcher J. 40- $\mathrm{Hz}$ oscillations during motor behavior in man. Neurosci Lett 1993;162:179-82.

23 Rushworth MFS, Ellison A, Walsh. Complementary localization and lateralization of orienting and motor attention. Nat Neurosci 2001;3:656-61.

24 Inoue T, Shimizu H, Nakasato N, et al. Accuracy and limitation of functional magnetic resonance imaging for identification of the central sulcus: comparison with magnetoencephalography in patients with brain tumors. Neuroimage 1999;10:738-48.

25 Cheyne D, Weinberg $\mathrm{H}$. Neuromagnetic fields accompanying unilateral finger movements: pre-movement and movement-evoked fields. Exp Brain Res 1989;78:604-12.

26 Taniguchi M, Yoshimine T, Cheyne D, et al. Neuromagnetic fields preceding unilateral movements in dextrals and sinistrals. Neuroreport 1998;9:1497-502

27 Ray WJ, Slobounov S, Mordkoff JT, et al. Rate of force development and the lateralized readiness potential. Psychophysiology 2000;37:757-65

28 Slobounov SM, Rearick MP, Simon RF, et al. Movement-related potentials area task or end-effector dependent: evidence from a multifinger experiment. Exp Brain Res 2000;135:106-16.

29 Singh KD, Barnes GR, Hillebrand A, et al. Task-related changes in cortical synchronization are spatially coincident with the hemodynamic response. Neuroimage 2002;16:103-14. 